\title{
La vida conectiva. Las redes digitales como espejos sociotécnicos de Iberoamérica
}

\author{
Pedro RODRIGUES COSTA \\ Universidade do Minho (Portugal) \\ pedrocosta@ics.uminho.pt

\section{Edson CAPOANO} \\ Universidade do Minho (Portugal) \\ edson.capoano@ics.uminho.pt
}

\section{Daniel BARREDO IBÁÑEZ}

Universidad del Rosario (Colombia); Fudan University (China) daniel.barredo@urosario.edu.co 


\title{
Resumen
}

La vida conectiva se ha acelerado a partir de 2020, momento en que inició la pandemia de covid-19, la cual contribuyó a intensificar algunos procesos de digitalización en marcha desde hacía décadas. Esta vida conectiva también presenta nuevos desafíos, como el capitalismo de vigilancia, la economía de la atención y los intelectos contingentes, formados por potentes algoritmos persuasivos. En este contexto, la edición de Chasqui número 147 se propone como un observatorio de cultura digital transnacional iberoamericana. Para ello, hemos seleccionado 10 artículos que revelan redes significativas, y en que se estudian los comportamientos de los usuarios; se mapean hábitos, usos y costumbres iberoamericanas en redes sociales; se describen formas de organización en el consumo, producción y circulación de contenidos; se analizan técnicas y contenidos utilizados para manipular información y opinión; se describen e identifican en formas de propagar noticias falsas y discursos de odio; y se presentan nuevas propuestas teóricas para entender a Iberoamérica a través del análisis y la lectura de campos como la big data, el machine learning, la Inteligencia Artificial, los algoritmos, o los sistemas de análisis y visualización de datos.

Palabras clave: redes sociales; Iberoamérica; inteligencia artificial; transformación digital

\begin{abstract}
Connective life has accelerated since 2020, when the covid-19 pandemic began, which contributed to intensify some digitization processes that have been underway for decades. This connective life also presents new challenges, such as surveillance capitalism, the attention economy, and contingent intellects, formed by powerful persuasive algorithms. In this context, the 147th edition of Chasqui is proposed as an Ibero-American transnational digital culture observatory. To do this, we have selected 10 articles that reveal significant networks, and in which user behaviors are studied; Ibero-American habits, uses and customs are mapped on social networks; Forms of organization in the consumption, production and circulation of content are described; techniques and content used to manipulate information and opinion are analyzed; describe and identify themselves in ways of spreading false news and hate speech; and new theoretical proposals are presented to understand Latin America through the analysis and reading of fields such as big data, machine learning, Artificial Intelligence, algorithms, or data analysis and visualization systems.

Keywords: social networks; Iberoamerica; artificial intelligence; digital transformation
\end{abstract}




\begin{abstract}
Resumo
A vida conectiva tem sido acelerada desde 2020 após as restrições sociais devido à pandemia de covid-19, contribuindo para a intensificação de alguns processos de digitalização que estavam em curso há décadas. Tal vida conectiva também apresenta novos desafios, como o capitalismo de vigilância, a economia da atenção e a criação de intelectos contingentes - algumas vezes manobrados por desinformação e estratégias de verdade à medida e quase sempre quitados por poderosos algoritmos persuasivos. Neste contexto, a 147 edição do Chasqui propõe um olhar sobre a cultura digital transnacional ibero-americana. Para isso, foram selecionados 10 artigos que revelam significativas redes da região, nos quais foram pesquisados comportamentos dos usuários; mapeados hábitos, usos e costumes ibero-americanos contidos nas redes sociais; descritas formas de organização no consumo, produção e circulação de conteúdos; analisadas técnicas e conteúdos para manipular informações e opiniões; identificadas e descritas notícias falsas e discurso de ódio; e apresentadas novas propostas teóricas para entender a América Latina por meio da análise e leitura de campos como big data, aprendizado de máquina, inteligência artificial, algoritmos, sistemas de análise e visualização de dados.
\end{abstract}

Palavras chave: redes sociais; Ibero-América; inteligência artificial; transformação digital.

\title{
1. Introducción. Las redes como espejos sociotécnicos
}

Vivimos en la era de las redes y las plataformas digitales, en una incuestionable importancia en la dinámica social, relacional, económica e informacional del mundo contemporáneo. Uno de los hitos más destacados de dichas dinámicas es el halo de convergencia entre los sistemas, los algoritmos, las pantallas, los contenidos y los formatos. El espacio Iberoamericano, como el mundo en general, no escapa a esta fuerza (Capoano \& Costa, 2021; Barredo, Rodrigues \& Hidalgo, 2021). La sociedad de la comunicación e información dio origen a las redes sociales digitales, materializando un objeto de estudio de interés para la sociología y para las Ciencias Sociales en su conjunto (Simmel,1983 [1908]; Elias, 1993; Granovetter, 1985; Latour, 2012).

La cuestión es que la organización social, incrementada por estas conexiones sociotécnicas digitales, altera las prácticas y las dinámicas de la opinión pública. A través del empoderamiento mediado por estas plataformas en línea, los grupos sociales alcanzan nuevas posibilidades y geometrías. Como sugería Elias (1993, p. 189), “cada pasaje de una organización de sobrevivencia predominante para otra es seguida de otro patrón de individuación”. En el caso concreto de las redes, hay un patrón de individuación con mapas biográficos de asociaciones que se reproducen en las pantallas (Costa, 2013), que sirven de sustrato para orientar el actor en su contingencia sociotécnica (Latour, 2012). Las redes, de hecho, 
aparecen enraizadas como las bases de un nuevo patrón de individuación, que necesita ser investigado.

Por ello, en conjunto con el establecimiento de un patrón mediado por varias plataformas de comunicación, se fortalece la necesidad de abordar el desarrollo de estudios permanentes sobre las redes -que se interconectan por estructuras comunes, como la lengua española y portuguesa, en nuestro caso. Así, es posible el desarrollo de un "trabajo común de cooperación científica, por todo el mundo lusófono” (Martins, 2019, p. 97) e hispánico (Forattini, 1997). Además, el imaginario común, consolidado en el espacio de los países iberoamericanos, encuentra en estas redes sociotécnicas la posibilidad de concentración y de agrupamiento de información, permitiendo la creación de una cartografía iberoamericana.

De ese modo, y siguiendo la estela de algunos trabajos colaborativos -como el que realiza el Grupo temático 19 Comunicación digital, redes y procesos, de la Asociación Latinoamericana de Investigadores de la Comunicación-, el objetivo de este monográfico fue generar una reflexión y una observación transversales del espacio iberoamericano en los ámbitos culturales, sociedades y de las redes sociales digitales, en un momento en que asistimos a un desplazamiento de las miradas desde los cielos, a las pantallas (Virilio, 2001). Y qué mejor hacerlo desde un enfoque amplio, colaborativo, característico de las tendencias y tradiciones investigativas propias de un contexto tan diverso en imaginarios, lenguas y tradiciones. El territorio sufre influencia por el ritmo de las pantallas y de Internet, "incluso por plataformas móviles de comunicación, información y ocio (iPads, tabletas, smartphones), movilizado también por nuevas formas de interacción social (entre las cuales las redes sociotécnicas) y por modelos emergentes de interacción (apps y videojuegos)" (Martins, 2019, p. 97), lo que hace inconcebible pensar en Iberoamérica (u otra región globalizada) sin tenerse en cuenta estas dinámicas reticulares.

\section{Desarrollo. Las redes sociotécnicas y la conexión iberoamericana}

Entre América y el Mediterráneo hay un reflejo que, como explica Carlos Fuentes en "Espejo Enterrado" (1992), alude a una historia política común entre ambos contextos, una cultura y unas trayectorias similares, particularmente entre la península ibérica y América Latina, que conlleva ecos y lazos estrechos que se mantienen todavía en el siglo XXI. Es, como describía Colombo (2001), un vínculo común que enlaza pasado y porvenir.

Para el reconocido escritor mexicano Carlos Fuentes, América Latina maduró al percibir el hecho de estar conformada por naciones multiculturales y no solo descendientes de europeos (como en la referencia que hizo el presidente argentino Alberto Fernández a la canción de Litto Nebbia "Llegamos de los Barcos", con la intención de evocar a la de Octavio Paz). Fuentes parte de una idea semejante a la de aquel escritor coterráneo ("los mexicanos descienden 
de los aztecas, los peruanos de los incas y los argentinos... de los barcos"), pero desarrolla una profecía más allá de nuestros orígenes, hacia un futuro común, con desafíos locales, hibridismos culturales y nuevas movilidades, como describe Colombo (2001), para quien el siglo XXI se caracterizará, precisamente, por el mestizaje y la importancia emergente de los grupos minoritarios.

Internet apenas había llegado en América Latina tres años antes del estreno de la serie "Espejo Enterrado" y de la publicación del libro homónimo, en el mismo México, a 28 de febrero de 1989, en el campus del Tecnológico de Monterrey (ITESM). Lo que Fuentes no podía adivinar es que, además de los espejos de obsidiana de El Tajín/Veracruz retratados en su libro, o los retratados por Velázquez y Cervantes, los iberoamericanos se verían en otros espejos, las pantallas conectadas en internet, hechas de cristal líquido. Hoy, nuestras sociedades son comprendidas no solo desde la reflexión de los espejos negros de Tezcatlipoca, sino también por las relaciones sociotécnicas desarrolladas en los últimos 20 años.

En lo que se refiere al campo de la comunicación y la información en línea, estamos en un momento de superación de los planteamientos dicotómicos que establecían una escisión entre las perspectivas utópicas e integradas, frente a las distópicas y apocalípticas. Dichos planteamientos se originaron en un momento iniciático en que la tecnología estaba avanzando hacia la integración personal y profesional; pero, en la actualidad, ya no es posible situar el debate entre la necesidad o no de la implementación de las redes sociotécnicas, dada la hipermediatización del individuo contemporáneo (Barredo, Rodrigues \& Hidalgo, 2021).

Más que discutir sobre la idoneidad o no de estas plataformas, que se han integrado en la cotidianidad global, se discuten los efectos sobre el consumo (Capoano et al., 2021a); se subraya la aparición de nuevos actores que, como las cibertropas, articulan campañas de desinformación (Bradshaw \& Howard, 2017); y se promueve la necesidad de innovar ciertos planteamientos desde el ámbito periodístico u organizacional (Barredo et al., 2020), en aras de una mayor participación, por citar algunos de los objetos de estudio recurrentes.

Los espejos sociotécnicos imprimen un reflejo de lo real, prolongan al individuo desde las nuevas posibilidades de colaboración, lo vinculan al colectivo, a los colectivos, convirtiéndose en aquellas redes esperanzadoras e indignadas que subrayaba Castells (2012) hace casi una década. Pero, también, estas plataformas pueden contribuir al desgaste del tejido social iberoamericano, aislando a los grupos en burbujas de pensamiento, en cámaras de eco que favorecen la polarización (Chenou et al., 2021), y que terminan erosionando a las democracias contemporáneas (Lupu, Ramírez \& Zechmeister, 2020), en favor de la emergencia de los autoritarismos populistas.

Esto es, los espejos conectados pueden ampliar la panorámica iberoamericana, ofrecer un repertorio de ideas, una transformación de las agendas, y una expansión de la diversidad. $\mathrm{O}$, por el contrario, pueden devolver 
el reflejo de un pensamiento concreto y, con ello, contribuir a profundizar la brecha entre unos países que presentan diferencias tan extremas.

Así como Carlos Fuentes, otros notables pensadores iberoamericanos supieron descifrar las imágenes reflejadas en nuestros espejos. El último que nos ha dejado, Jesús Martín-Barbero, a 12 de junio de 2021, se transformó él mismo en parte de su obra, al volverse un cartógrafo para los caminos y rumbos de la cultura y la comunicación latinoamericanas. Fue Martín-Barbero quien consolidó la importancia de la mediación en el proceso comunicativo, indisociable de las relaciones y del proceso de recepción informativo (MartínBarbero, 1987; 1997, 2010).

En nuestros actuales espejos conectados, la mediación de la tecnología también asume un peso fundamental para la composición de la cultura producida en las redes sociotécnicas. Sin embargo, dicho proceso no define por sí solo las relaciones entre individuos, y entre éstos y la política, la economía y el territorio, sino que las redefine, a través de la socialidad, la ritualidad y la tecnicidad (Martín-Barbero, 2010). Pese que nos asombre la perspectiva posible de las acciones humanas controladas por los algoritmos y la big data, como se señala en obras audiovisuales como "The Social Dilemma” (JeffOrlowsky, Netflix, 2020) y "Black Mirror", (Charlie Brooker, Netflix, 2001-2019), la responsabilidad de la construcción de sociedades mejores con el uso de las redes sociotécnicas -y aunque parezca utópico-, sigue en nuestras manos.

\section{La vida conectiva: dilemas y desafíos}

La vida conectiva se ha acelerado a partir de 2020, momento en que inició la pandemia de covid-19, la cual contribuyó a intensificar algunos procesos de digitalización en marcha desde hacía décadas. En Portugal, por ejemplo, con 8,52 millones de usuarios de internet y cerca de 10 millones de habitantes, y una tasa de penetración de la red de cerca del 78\% en enero de 2020 (Newman et al., 2021), se evidenció un crecimiento del $36 \%$ en el consumo de videos cortos de hasta 15 segundos en la plataforma TikTok, un mes después del inicio de la pandemia (en abril). En marzo del mismo año, hubo un aumento de un 115\% en las vistas de videos clasificados como noticias y política en plataformas de videos de Internet como YouTube, Facebook o Instagram. Durante el mismo año, el uso de la televisión en plataformas web como Netflix creció un 31,7\% (Hootsuite, 2021). Estos usos, costumbres y prácticas análogas en las redes y plataformas digitales demuestran el crecimiento de lo que llamamos vida conectiva en prácticamente todos los ámbitos de lo individual y colectivo.

Esta vida conectiva también presenta nuevos desafíos, como el capitalismo de vigilancia, la economía de la atención y los intelectos contingentes, formados por potentes algoritmos persuasivos (Barredo, 2021; Bradshaw \& Howard, 2017; Costa, 2020a; 2021a). El primer fenómeno surge con la dinámica de apropiación y monetización de datos por parte de grandes corporaciones 
y empresas de tecnología, que recopilan y estructuran información y datos digitales para obtener ganancias (Zuboff, 2016). El segundo, por su parte, resulta del capitalismo de vigilancia, ya que los datos generados por la huella digital del sujeto permiten el registro de información y de datos por las empresas digitales. De hecho, dichas organizaciones desarrollan estrategias de "seducción” y "persuasión" para captar la atención, como el uso de arquetipos seductores en los procesos de edición de videos en YouTube (Costa, 2020a), o a través de técnicas de laboratorios conductuales o de juegos que favorecen la adicción y el hiperconsumo (Costa, 2020b; Patino, 2019). Aunque hay algoritmos pensados para ayudar a la vida conectiva -como en el caso de los bots diseñados para dar respuesta a un servicio concreto, como explica Barredo (2021) -, a su vez, los algoritmos difunden ideas, opiniones, emociones, desinformación y/o promocionan a quienes más influyen en las sociedades contemporáneas (Capoano \& Costa, 2021a; Costa, 2021a; 2021b). Sin embargo, hay ejemplos de dinámicas sociotécnicas que benefician a esos grupos que generalmente están en desventaja, como los grupos minoritarios que utilizan las redes para equipararse a los grandes players de la comunicación. Este es el caso de la dinámica periodística iniciada por Mural, agencia de periodismo de la periferia de São Paulo que informa sobre las favelas, ofreciendo historias sobre la vida de las afueras a través de las redes digitales (Capoano et al, 2021b).

Ya en una perspectiva individual, la vida conectiva revela efectos y desafíos que afectan a las personas más conectadas, movilizadas y propensas a dinámicas generadoras de hábitos, efectos psicosociológicos adictivos y problemas relacionales. Es el caso del efecto tragamonedas -que parte de la conocida teoría de usos y gratificaciones (Katz, 1959)-, es decir, el estímulo de la adicción desde la invitación permanente a recibir recompensas aleatorias contenidas en el simple gesto de actualización con el scroll-down de las plataformas digitales (Costa, 2020); el caso del efecto Zeigarnik, un conjunto de acciones encadenadas y enlazadas sin pausas, generando sensación de vacío a través de la dosificación sutil de satisfacciones y frustraciones; el efecto de plenitud, utilizado en el sistema "auto-play" de plataformas streaming como YouTube, Netflix o HBO, en que la frustración asociada con la visualización incompleta de un contenido es compensada por una cadena de opciones automáticas que genera dependencia, no sea interrumpida por otras demandas.

De lo anterior se desprende la existencia de una planificación que, en vez de favorecer la expansión y el enriquecimiento del pensamiento, de la colaboración entre los usuarios, se apropia de la vida conectiva a través de unos algoritmos que deciden por el sujeto y que se orientan a captar su acción y atención (Patiño, 2019). Otro de los efectos perniciosos es el efecto de los centinelas dormidos, una patología que hace que los sujetos no duerman profundamente por temor a perderse una notificación del smartphone (Patino, 2019). Este efecto agrega cambios significativos al estado de ánimo debido a la reducción del tiempo de sueño y descanso, la dificultad en la atención y la concentración 
(Eisenstein \& Estefanon, 2011); o el FOMO - acrónimo de "fear of missing out" o miedo a perderse algo, como cuando el sujeto está sin Internet (Przybylski et al., 2013); la Nomophobia - acrónimo de "No Mobile Phobia”, el pánico de estar sin teléfono o de alejarse del smartphone (Patiño, 2019, p. 20); el Phubbing, acrónimo de "Phone" (teléfono) y "Desaire" (despreciar)-, que consiste en la verificación ostensible del móvil mientras alguien está interactuando presencialmente, efecto que ha empobrecido las interacciones sociales (Costa, 2020); o, entre otros, el miedo al consumo solitario, detectado en comentarios de jóvenes cuando disfrutan de vídeos en plataformas de streaming de vídeo, como YouTube o VeVo, sin interlocución de sus pares. Este disgusto se nota principalmente en las generaciones $\mathrm{Y}$ y $\mathrm{Z}$, dadas sus características de consumo de infoentretenimiento, realizado en plataformas sociales digitales, de forma colectiva. Es una nueva forma de probar y afrontar la formación de la identidad en su relación entre iguales (Costa \& Capoano, 2020).

\section{Conclusiones: las redes digitales como espejos sociotécnicos, la temática central del número}

Como vemos, la vida conectiva es un trasunto dinámico, enriquecido a partir de los distintos avances sociotécnicos. En este sentido, este monográfico tuvo como objetivo convocar a artículos iberoamericanos centrados en examinar las redes sociales digitales desde una perspectiva panorámica, con los efectos que las relaciones sociotécnicas (Latour, 2012) tienen sobre los individuos, las comunidades y las sociedades. Pues la infinidad de la web dispersa el trabajo y los resultados, provocando un caos informativo en este campo investigativo, organizado por criterios de búsqueda difusos -como las palabras clave de indexación-, los intereses de los usuarios, los algoritmos y los mecanismos de focalización pagados, con fines promocionales.

En este contexto, propusimos constituir nuestro número como un observatorio de cultura digital transnacional iberoamericano. Creemos que lo hemos logrado: los artículos de este monográfico revelan redes significativas, trabajos que tratan con otras regiones de forma conectada y polos sociotécnicos en el espacio cultural lusófono e hispánico.

En total, y a través de un riguroso proceso de evaluación por pares, se han seleccionado 10 artículos que ofrecen tanto planteamientos más amplios y reflexivos, como resultados concretos desde los estudios de caso sobre algunos de esos fenómenos que identifican a la opinión pública iberoamericana contemporánea. Así, en el primer artículo, Carla Cándida Rizzotto y Luciane Leopoldo Belin presentan un estudio de 3207 comentarios publicados en las páginas de Facebook de tres medios. Las autoras encuentran un predominio de los comentarios racionales, así como las experiencias y los valores personales. En el segundo artículo, María Beatriz Juárez Escribano y Lidia Mañoso Pacheco se cuestionan la manipulación de la identidad y los perfiles virtuales en las 
redes sociales. Sobre este punto, las autoras subrayan que unos 8 de cada 10 individuos no son sinceros con el grupo al que se dirigen, en tanto que tienden a mantener oculta su identidad real. Alexandre Duarte y Patrícia Dias, en el tercer artículo, estudian los usos y las motivaciones de los adolescentes portugueses sobre una de las plataformas más pujantes en este momento, como es TikTok. En este medio social - que fue el más descargado durante la pandemia de covid19 - predominan, de acuerdo a los autores, los contenidos pensados de forma estratégica, esto es, los contenidos autopromocionales.

En el cuarto artículo, Vítor de Sousa presenta un estudio de caso sobre la identidad transnacional y transcultural asociada al Museo Virtual da Lusofonía, que puede ser un centro de interés, como explica el autor, para generar un punto de encuentro intercultural. Raul Anthony Olmedo Neri, en el quinto artículo, analiza el movimiento LGBT desde el estudio de un hashtag o tendencia. A través de la técnica del Análisis de Redes Sociales, el autor encuentra la existencia de comunidades conectadas, no tan interactivas con otras comunidades.

En el sexto artículo, Pavel Sidorenko, Francisco Cabezuelo y José María Herranz de la Casa estudian el caso de Pictoline (México) dentro de lnstagram, como un instrumento adaptado para la difusión del conocimiento científico. Los autores constatan el rol copartícipe de los usuarios, en conjunto con su participación activa para favorecer una apropiación de los contenidos. Antonio Helio Junqueira, Rodrigo Eduardo Botelho y Jenifer Daiane Greger, en el séptimo artículo, reflexionan sobre las vulnerabilidades digitales, desde una sistematización bibliográfica, bajo una perspectiva crítica. Los autores, en este artículo de revisión, relacionan los abordajes nacionales e internacionales sobre esta temática, que es muy pertinente para entender las consecuencias de las redes sobre las sociedades a las que se dirigen.

En el octavo artículo, Lia Gabriela Pagoto y Raquel Ritter Longhi examinan la deslegitimación del periodismo a partir de las redes sociotécnicas y, en concreto, mediante las alocuciones del presidente Jair Bolsonaro a sus usuarios. $\mathrm{Al}$ analizar los comentarios publicados en CNN Brasil y Folha de Sao Paulo, las autoras encuentran una correlación entre el planteamiento de los usuarios con los ataques del presidente brasileño. Por su parte, Eliete da Silva Pereira y Massimo Di Felice, en el noveno artículo, estudian las redes sociotécnicas a partir de tres experiencias relacionadas con los indígenas de Brasil. Desde un enfoque cualitativo, los autores observan la estructura de estas redes y encuentran una interacción anclada a temas ecológicos, ambientales y cosmológicos. Por último, María Mendoza Michilot, en el décimo artículo, se centra en analizar la interacción de los usuarios peruanos alrededor de un conflicto socioambiental en ese país. La autora describe los vínculos entre los usuarios derivados de la discusión virtual, así como la valoración de los protagonistas que formaron parte del mismo.

En definitiva, es este un número que, al estudiar las tecnologías de la informaciónylacomunicación desdeladiversidadmetodológicayepistemológica 
iberoamericana, ofrece un mapa de circunnavegación tecnológica -por seguir la sugerencia de Martins (2019) - que articula el sentido de comunidad cultural a través de las redes de conocimiento transculturales y transnacionales. Esta transnacionalidad de la investigación iberoamericana resiste al aislamiento de la ciencia impuesto por el idioma inglés frente al español y al portugués. Como condición tecnológica de nuestro tiempo, la cultura y las artes también se extienden por redes sociotécnicas, pasando a la opinión pública digital (Kerckhove, 1997), junto con la aparición de una constelación de territorios y paisajes: sitios web, portales, blogs, juegos, repositorios y museos virtuales, entre otros.

Los artículos seleccionados corresponden a la centralidad de estos ejes temáticos. Se recopiló, para ello, información sobre las redes sociales digitales utilizadas por las comunidades en Iberoamérica para actualizar los estudios de recepción; se mapean hábitos, usos y costumbres iberoamericanas en redes sociales; se describen formas de organización en el consumo, producción y circulación de contenidos; se analizan técnicas y contenidos utilizados para manipular información y opinión; se describen e identifican formas de propagar noticias falsas y discursos de odio; y se presentan nuevas propuestas teóricas para entender Iberoamérica a través del análisis y lectura de campos como la big data, el machine learning, la Inteligencia Artificial, los algoritmos, o los sistemas de análisis y visualización de datos.

Así, con este número 147 de Chasqui, se logró uno de los objetivos fundamentales: la generación de un diálogo entre el conocimiento teórico y empírico en el espacio iberoamericano entre la teoría de las redes sociales (Tarde, 1992 [1901]; Simmel, 1983 [1908]; Elias, 1993; Granovetter , 1985; Higgins y Ribeiro, 2018), la teoría del Actor-Red y la dimensión sociotécnica de la acción (Law, 1992; Callon, 1998; Latour, 2012), la teoría de los espejos aplicada al universo del ciberperiodismo y las prácticas informativas del ciberactivismo (Lippmann, 1922; Pena, 2010), la teoría de los ecos y las resonancias del lienzo en las dimensiones de la inteligencia emocional y social (Mayer, Salovey y Caruso, 2008; Simondon, 1989; Costa, 2013), las dinámicas generadas por el aprendizaje automático y la inteligencia artificial (Langley, 2011; Mitchell, 1997; Harnad, 2008; Russell y Norvig, 2003), y el análisis de la big data (Friedman, 1998; Simon, 2013); junto con las reflexiones sobre la decolonialidad y la comunicología en los ambientes digitales (Huérfano, Sierra Caballero y del Valle Rojas, 2016). Un gran trabajo colaborativo, que muestra el dinamismo de la investigación iberoamericana. Disfrútenlo.

\section{Referencias}

Barredo Ibáñez, D. \& Díaz Cerveró, E. (2017). La interactividad en el periodismo digital latinoamericano. Un análisis de los principales cibermedios de Colombia, México y Ecuador (2016). Revista Latina de Comunicación Social, 72, 273-294. 
Barredo Ibáñez, D. (2018). Religious Commitment, Subjective Income, and Satisfaction towards the Functioning of Democracy in Latin America. A Mediation Analysis Model Based on Latinobarómetro. Religions, 9, 1-12. Link: http://www.mdpi.com/2077$1444 / 9 / 6 / 198$

Barredo Ibáñez, D. (2021). Medios digitales, participación y opinión pública. Bogotá: Tirant Lo Blanch.

Barredo Ibáñez, D. B., Cunha, M. R. \& Toledo, J. H. (2021). Comunicación digital, redes sociales y procesos en línea: estudios en una perspectiva comparada entre América Latina y la península ibérica. Journal of Iberian and Latin American Research, 26(3), 275-283. https://doi.org/10.1080/13260219.2020.1934260

Barredo Ibáñez, D.; Pinto Garzón, K. T.; Freundt-Thurne, Ú. \& Medranda, N. J. (2020). Interaction and user- generated content on online informative platforms: A comparison of journalists in Colombia, Peru and Ecuador. Catalan Journal of Communication \& Cultural Studies, 12(1), 99-117. https://doi.org/10.1386/cjes_00o16_1

Barredo, D.; Rodrigues, M; Hidalgo, J. A. \& La Rosa, A. (Eds.) (2019). Movilización ciudadana, medios sociales e Internet: miradas latinoamericanas. La Laguna, España: Sociedad Latina de Comunicación Social. ISBN: 978-84-17314-23-1. Link: http://www.cuadernosartesanos.org/2019/cac165.pdf

Bradshaw, S. \& Howard, P. N. (2017). Troops, Trolls and Troublemakers: A Global Inventory of Organized Social Media Manipulation. University of Oxford Working Paper No. 2017.12. Available at: https://comprop.oii.ox.ac.uk/wp-content/uploads/sites/89/2017/o7/TroopsTrolls-and-Troublemakers.pdf

Callon, M. (1998). El proceso de construcción de la sociedad: el estudio de la tecnología como herramienta para el análisis sociológico. In: Doménech, M. \& Tirado, F. J. (Eds.) Sociologia simétrica. Barcelona: Gedisa.

Capoano, E. \& Costa, P. R. (2021). Emotions, morals and resilience: the consumption of news during the Covid-19 pandemic. In Berube, D. (2021). Pandemic Communication. New York: Springer. ISBN: 978-3-030-77343-4.

Capoano, E., Costa, P. R., Barros, V., Galhardi, R. (2021a). Tristeza, medo, raiva e vergonha: das emoções ao neuroticismo estimulados pelo consumo de notícias sobre Covid-19. In Oliveira, R. C., Christino, D. \& Júnior, E. V. M. (Organizadores). Covid-19 e a Comunicação: 254-277. Goiânia: Cegraf UFG. ISBN: 978-65-89504-64-1.

Capoano, E., Rovida, M.; Alencar, V. (2021). Identidades, perfiles, productos y procesos: elementos que componen la Agencia Mural de periodismo de las periferias. In La revolución de los prosumers: youtubers e instagramers, Editora Egrerius. Huelva, España. ISBN 978-84-18167-56-0.

Castells, M. (2012). Redes de Indignación y Esperanza. Madrid: Alianza Editorial

Chenou, J. M.; Cabarcas, D. \& Sepulveda, M. N. (2021). "Social media and political polarization in Latin America. Analyzing online discussions during the 2018 presidential campaign in Colombia”. In Ramírez, D.; Carvalho, B. \& Plaw, A. (Eds.). The Politics of Technology in Latin America (vol. 2) <pp. 129-146>. New York: Routledge.

Colombo, Sylvia (2001). Literatura: Carlos Fuentes constrói ponte sobre o Atlântico. Folha de S. Paulo. https://www1.folha.uol.com.br/folha/ilustrada/ult9ou13889.shtml

Costa, P. R. \& Capoano, E. (2021). O medo do consumo solitário: comentários em canais infantojuvenis de YouTube do Brasil e de Portugal. Journal of Iberian and Latin American Research, 26(3), 407-426, https://www.tandfonline.com/doi/full/10.1080/1326021 9.2020 .1909872 
Costa, P. R. (2013). Entre o ver e o olhar: ecos e ressonâncias ecrãnicas. Braga: Universidade do Minho. Retirado de http://repositorium.sdum.uminho.pt/handle/1822/244.92

Costa, P. R. (2020a). A presença de arquétipos nos youtubers: modos e estratégias de influência. Revista Galáxia, 45: 5-19. https://doi.org/10.1590/1982-25532020347613

Costa, P. R. (2020b). Impactos da captologia. Problemáticas, desafios e algumas consequências do "dar vistas" ao ecrã em rede. Sociologia online, 23 (74-94). https://doi.org/10.30553/ sociologiaonline.2020.23.4

Costa, P. R. (2021a). Da ferramenta ao intelecto algorítmico: sobreviver entre dilemas digitais. Journal of Digital Media \& Interaction, 4 (10), 21-37. Disponível em https://proa.ua.pt/ index.php/jdmi/article/view/24568?fbclid=IwAR1-byFGnACBTOcYaiAeIkYQglhJHyzStUtxkuxgWiiP4qQfIqQb9I46eQ8

Costa, P. R. (2021b). O ethos wikipedista como modo de combate à desinformação. Liinc Em Revista, 17(1), e563o. https://doi.org/10.18617/liinc.v17i1.5630

Elias, N. (1993). O Processo Civilizador: formação do estado e civilização. Rio de Janeiro: Zahar.

Forattini, O.P. (1997), A língua franca da ciência. Saúde Pública, 31:3-8.

Friedman, J. H. (1998). «Data Mining and Statistics: What's the connection?». Computing Science and Statistics $29(1): 3-9$

Fuentes, C. (1992). El espejo enterrado. Colección Tierra Firme, Fondo de Cultura Económica, México.

Granovetter, M. (1985). Economic action and social structure: the problem of embeddedness. American Journal of Sociology (91). pp. 481-510.

Harnad, S. (2008). The Annotation Game: On Turing (1950) on Computing, Machinery, and Intelligence. in Epstein, Robert; Peters, Grace: Parsing the Turing Test: Philosophical and Methodological Issues in the Quest for the Thinking Computer (em inglês). [S.l.]: Springer. pp. 23-66. Consultado em 10 de outubro de 2016.

Herrera Huérfano, E., Sierra Caballero, F., \& Del Valle Rojas, C. (2016). Hacia una Epistemología del Sur. Decolonialidad del saber-poder informativo y nueva Comunicología Latinoamericana. Una lectura crítica de la mediación desde las culturas indígenas.

Higgins, S. S., \& Ribeiro, A. C. (2018). Análise de redes em ciências sociais. Brasília: ENAP.

Hootsuite (2021). The Global State of 2021. Digital Trends 2021. Hootsuite. We are Social. Disponível em https://www.hootsuite.com/resources/digital-trends

John D. Mayer, Peter Salovey and David R. Caruso (2008). "Emotional Intelligence: New Ability or Eclectic Traits?”. American Psychologist, Vol. 63, No. 6, pages 503 - 517.

Katz, E. (1959). Mass Communications Research and the Study of Popular Culture: An Editorial Note on a Possible Future for This Journal. Studies in Public Communication, 2, 1-6. Recuperado de: http://repository.upenn.edu/asc_papers/165

Kerkhove, D. (1997). A pele da cultura. Lisboa: Relógio D’água.

Langley, P. (2011). «The changing science of machine learning». Machine Learning (em inglês). 82 (3): 275-279. doi:10.1007/s10994-011-5242-y. Consultado em 10 de outubro de 2016

Latour, B. (2012). Reagregando o Social - Uma Introdução à Teoria do Ator-Rede. Salvador: Edusc.

Law, J. (1992). Notes on the Theory of the Actor-Network: Ordering, Strategy and Heterogeneity. Systems Practice, 5 (4), 379-393. Disponível em: http://www.lancs.ac.uk/fss/ sociology.

Lippmann, W. (1922). Public Opinion. New York: Free Press. 
Lupu, N.; Ramírez, M. V. \& Zechmeister, E. J. (2020). Social Media Disruption: Messaging Mistrust in Latin America. Journal of Democracy, 31(3), 160-171. Available at: https:// muse.jhu.edu/article/76008o/summary

Martin-Barbero, Jesús (1987). De los medios a las mediaciones: comunicación, cultura y hegemonía. Barcelona: Gustavo Gili, 1987.

Martin-Barbero, Jesús (1997). Dos meios às mediações: comunicação, cultura e hegemonia. Rio de Janeiro: UFRJ, 1997.

Martin-Barbero, Jesús (2003). Oficio de cartógrafo. México: Fondo de cultura económica.

Martin-Barbero, Jesús (2010). De los medios a las mediaciones: comunicación, cultura y hegemonía. De los medios a las mediaciones, 1-335.

Martins, M. L. (2019). O português é uma língua não só de comunicação, mas também de culturas, pensamento e conhecimento. Matrizes, (13), 1. São Paulo: Brasil.

Mitchell, T. (1997). Machine Learning. [S.l.]: McGraw Hill. 2 páginas. ISBN 0-07-042807-7

Newman, N. Fletcher, R., Schulz, A., Andı S., Robertson, Graig, Nielsen, R. K. (2021). Digital News Report 2021 (1oth edition). Reuters Institute for the Study of Journalism. Reuters Institute, Oxford University. Disponível em https://reutersinstitute.politics.ox.ac.uk/ sites/default/files/2021-06/Digital_News_Report_2021_FINAL.pdf

Patino, B. (2019). A civilização do peixe-vermelho: Como peixes-vermelhos presos aos ecrãs dos nossos smartphones. Lisboa: Gradiva.

Pena, F. (2010). Teoria do Jornalismo. São Paulo: Contexto.

Przybylski, A., Murayama, K., DeHaan, C., \& Gladwell, V. (2013). Motivational, emotional, and behavioral correlates of fear of missing out. Computers in Human Behavior, 29(4), 1841-1848. doi: 10.1016/j.chb.2013.02.014

Russell, S.; Norvig, P. (2003 [1995]). Artificial Intelligence: A Modern Approach.

Simmel, G. (1983/1908). Sociologia. São Paulo: Ática S.A.

Simon, P. (Março, 18, 2013). Too Big to Ignore: The Business Case for Big Data. [S.l.]: Wiley. 89 páginas.

Simondon, G. (1989). Du mode d'existence des objets techniques. Paris: Aubier.

Tarde, G. (1992/1901). A Opinião e as Massas. São Paulo: Martins Fontes.

Virilio, P. (2001). Entretien avec Paul Virilio. Le Monde de L'Education, 294: 135-138.

Zuboff, V. S. (2016, 5 de março). The Secrets of Surveillance Capitalism. In Frankfurter Allgemeine. Retirado de https://www.faz.net/aktuell/feuilleton/debatten/the-digital-debate/ shoshana-zuboff-secrets-of-surveillance-capitalism-14103616.html?printPagedArticle=true 
